\title{
IL13Ra1 protects against rheumatoid arthritis by combating the apoptotic resistance of fibroblast-like synoviocytes
}

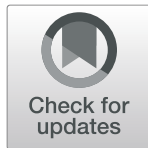

Xiaomei Yang ${ }^{1,2}$, Qingwei Guo ${ }^{1}$, Tingting Feng ${ }^{3}$, Qiqi Lu, ${ }^{4,5}$ Luna Ge${ }^{4}$, Jihong Pan ${ }^{4}$, Kehong Bi ${ }^{2}$, Li Qiao ${ }^{6}$, Lei Tian ${ }^{6}$, Tianhua Xie ${ }^{7}$, Chengfang $\mathrm{YaO}^{8}$, Guanhua Song ${ }^{8^{*}}$ and Lin Wang ${ }^{4^{*}}$

\begin{abstract}
Background: Endoplasmic reticulum (ER) stress is closely related with the pathological progression of rheumatoid arthritis (RA), and fibroblast-like synoviocytes (FLSs) are known as its resistance against ER stress-induced apoptosis. Studies on overcoming such resistance would provide a novel treatment strategy for RA in a clinical setting.

Methods: IL13Ra1 expression was assessed in the synovial tissue by RT-qPCR, immunohistology, and Western blot. Gain or loss of functional analysis was applied to evaluate the biological roles of IL13Ra1 in RA FLSs. Cell viability and apoptosis were assessed by MTS, Western blot, and flow cytometry. The therapeutic effects of IL13Ra1 on the severity of type II collagen-induced arthritis (CIA) in DBA-/1 mouse model were evaluated by scoring synovitis, hyperplasia, cartilage degradation, and bone destruction.

Results: IL13Ra1 expression was selectively downregulated when RA FLSs were stimulated by ER stress inducers. Functionally, IL13Ra1 overexpression could inhibit the viability, but induce the apoptosis of RA FLSs in the presence of ER stress inducers. Mechanistically, IL13Ra1 promotes cell apoptosis via transcriptionally activating trail expression. Besides, IL13Ra1 could interact and stabilize DR5 protein, thus forming a positive loop involving trail and DR5 to render RA FLSs more susceptible to apoptosis. Additionally, intraarticular injection of IL13Ra1 conferred therapeutic effects in CIA models and showed a limited degree of synovial proliferation and joint destruction.
\end{abstract}

Conclusions: Together, our data establishes a regulatory role for IL13Ra1 to combat the apoptotic resistance of RA FLSs against ER stress. The inhibitory effects of IL13Ra1 on arthritis progression suggest the therapeutic potential in RA.

Keywords: Rheumatoid arthritis, ER stress, IL13Ra1, Apoptosis, Synoviocyte

\footnotetext{
*Correspondence: yysghyy@163.com; kecheng1216@163.com

${ }^{8}$ Institute of Basic Medicine, Shandong First Medical University \& Shandong

Academy of Medical Sciences, Jinan 250062, China

${ }^{4}$ Department of Rheumatology and Autoimmunology, The First Affiliated Hospital of Shandong First Medical University, Key Lab for Biotech-Drugs of National Health Commission, Key Lab for Rare \& Uncommon Diseases of

Shandong Province, Shandong Medicinal Biotechnology Centre, Jinan 250062, China

Full list of author information is available at the end of the article
}

(c) The Author(s). 2020 Open Access This article is licensed under a Creative Commons Attribution 4.0 International License, which permits use, sharing, adaptation, distribution and reproduction in any medium or format, as long as you give appropriate credit to the original author(s) and the source, provide a link to the Creative Commons licence, and indicate if changes were made. The images or other third party material in this article are included in the article's Creative Commons licence, unless indicated otherwise in a credit line to the material. If material is not included in the article's Creative Commons licence and your intended use is not permitted by statutory regulation or exceeds the permitted use, you will need to obtain permission directly from the copyright holder. To view a copy of this licence, visit http://creativecommons.org/licenses/by/4.0/ The Creative Commons Public Domain Dedication waiver (http://creativecommons.org/publicdomain/zero/1.0/) applies to the data made available in this article, unless otherwise stated in a credit line to the data. 


\section{Introduction}

Endoplasmic reticulum (ER) stress is a common cellular response in rheumatoid arthritis (RA) [1, 2]. Hypoxia, glucose deprivation, reactive oxygen species, and proinflammatory cytokines could induce ER stress, thereby provoking the excessive accumulation of unfolded protein in both innate immune cells (e.g., dendritic cells, adaptive immune cells (e.g., $\mathrm{T}$ and $\mathrm{B}$ cells), and mesenchymal stromal cells (fibroblast-like synoviocytes (FLSs)) in the inflamed joints [3]. Although unfolded protein response (UPR) can alleviate sustained ER stress by inhibiting protein translation, activating chaperones, and enhancing proteasome degradation of misfolded proteins, the persistent ER stress will turn on the proapoptotic arm of UPR via inducing ChoP expression [4]. Several studies have highlighted the importance of ER stress as its effects on cellular apoptosis, proliferation, inflammation, and their potential consequences for synovial hyperplasia [5, 6]. RA FLSs produce large amounts of cytokines and enzymes, and $30 \%$ of all newly synthesized, ER-sorted proteins are unfolded [1, 3, 5]. However, unlike other cell types, RA FLSs are resistant to ER stress-induced apoptosis and the mechanistic basis underlying for it remains still unclear [7]. Identifying local factors that are able to combat this resistance is therefore of great importance to understand the pathological characters and also potential intervention for RA.

Numerous studies reported that IL-13could be viewed as a serum biomarker and therapeutic target for RA [8-11]. Besides its anti-inflammatory role, IL-13 can also protect human synovial cells from apoptosis and promote synovial proliferation $[5,12]$. IL13R $\alpha 1$ is required for delivering IL13 responses of fibroblasts, eosinophils, and dendritic cells to affect the pathological features associated with asthma and lung injury [8]. Upon binding to IL-13, IL13R $\alpha 1$ forms a heterodimer with IL-4R $\alpha$, internalizes into the cytosol and consequently activates cytosolic signal transducer and activator of transcription 6 (STAT6) [9]. Importantly, it has been reported that IL-13 could induce ER stress and promote apoptosis and death in microglia [13]. Furthermore, ER stress can impair IL-13 signaling via repressing IL4R $\alpha$ [14]. However, the precise roles of its receptors in the cellular response towards ER stress are still unclear in RA. In addition, IL13R $\alpha 1$ has no intrinsic kinase activity but rather constitutively associates Tyk2 via its proline-rich Box-1 region in the cytoplasm [8]. Intriguingly, the increased pathology of pulmonary injury in bleomycin-treated IL13R $\alpha 1^{-/-}$ mice may be largely independent of IL-13 [12]. Hence, it is important to define the activity and the signaling pathway downstream of IL13R $\alpha 1$ under ER stress.

Trail has several receptors include the agonistic receptors DR4 and DR5 [15-17], the decoy receptors Trail-R3 (DcR1) and Trail-R4 (DcR2), which antagonize the death signal $[18,19]$, and osteoprotegerin (OPG) [20]. DR5
(Trail-R2) was highly expressed in the synovial tissue and fibroblasts from RA and specific activation of TrailDR5 signaling was effective for ameliorating the arthritis phenotype of RA [21]. However, the regulatory mechanism towards Trail-DR5 remains largely unknown in RA FLSs.

In this study, we found that RA FLSs with IL13R $\alpha 1$ overexpression are more susceptible to apoptosis in response to ER stress. More importantly, the proapoptotic feature of IL13R $\alpha 1$ is also functional even in the absence of its ligands IL-13. At the molecular level, IL13R $\alpha 1$-mediated activation of STAT6 transcriptionally upregulates trail expression. Meanwhile, IL13R $\alpha 1$ interacts and stabilizes DR5 protein on the cell surface of RA FLSs. Therefore, IL13R $\alpha 1$ activates the Trail-DR5 signaling pathway and promotes apoptosis of RA FLSs. We also explore the therapeutic potential of IL13R $\alpha 1$ and demonstrate that IL13R $\alpha 1$ delivery into joint ameliorates the severity of collagen-induced arthritis (CIA).

\section{Materials and methods \\ Patients}

Human synovial tissues were collected from a total of 26 RA patients fulfilling the 1987 criteria of the American College of Rheumatology [22] and obtained at the time of joint replacement surgery at the Shandong Provincial Hospital and Shandong Qiaofo Mountain Hospital (Jinan, China). Twelve osteoarthritis (OA) patients [23] who underwent total joint replacement. The mean age of the RA patients ( 12 males and 14 females) was $54.8 \pm$ 10.9 years. The mean disease duration was 62.3 mo (range 9-159 months). The mean ages of the OA patients were $54.7 \pm 7.8$ years. The majority of RA patients were treated with disease-modifying antirheumatic drugs (DMARDs), including methotrexate, and none were on biologics. Demographic data are provided in Supplementary Table 1 . The study protocol was approved by the Institutional Review Board of Shandong Medicinal Biotechnology Center (VCMC08BR067). All patients gave fully informed written consent approved by the institutional ethics committee, and research was performed in accordance with the Declaration of Helsinki.

\section{Reagents and cell stimulation}

RA FLSs were cultured overnight to reach $80 \%$ confluence in medium containing $1 \%$ fetal cells and subsequently stimulated with the following reagent. Cycloheximide (CHX, $10 \mu \mathrm{g} / \mathrm{mL}, 239,763)$ was purchased from Calbiochem. Thapsigargin (Tg, T9033) and Tunicamycin (Tm, 654,380) were classical inducers for ER stress and purchased from Sigma. Cobalt chloride $\left(\mathrm{CoCl}_{2}\right)$ was bought from Sigma Company. STAT6 inhibitor (100 nM, AS1517499) and IL13 (213-ILB) were purchased from Selleck and R\&D Systems, respectively. 


\section{Cell isolation and culture}

FLSs were obtained from the synovial tissues of RA or OA patients as described previously [2] and cells between passages 4 and 7 were used for further study. Cells were cultured in Dulbecco's modified Eagle's medium (DMEM) with $10 \% \mathrm{FBS}$ in a $37^{\circ} \mathrm{C} 5 \% \mathrm{CO}_{2}$ incubator (Thermo, USA). To mimic the hypoxic condition, RA FLSs were treated with $\mathrm{CoCl}_{2}$ as described before [24].

\section{Lentiviral transduction}

Myc-tagged-wild type human and mouse IL13R $\alpha 1$ (wt) was constructed and cloned into pcDNA3.1/Zeo(+) (Thermo Fisher Scientific) by Cyagen Biosciences (Guangzhou, China). Lentivirus products were constructed in HEK293T cells by co-transfection with psPAX2, pMD2.G, and lentiviral construct (pLentiCMV/TO Puro DEST) expressing wild type IL13R $\alpha 1$ (Lv-IL13R $\alpha 1)$ using lipofectamine 2000 (Invitrogen, Carlsbad, CA, USA).

\section{siRNA transfection and apoptosis}

RA FLSs $\left(2 \times 10^{5}\right.$ cells in 100 -mm-diameter dishes or $8.5 \times 10^{4}$ cells in 6-well plates) were plated to reach $75 \%$ confluence and were transiently transfected with IL13R $\alpha 1$ or DR5 small interfering RNA (siRNA, Ruibo Biosciences, Guanzhou, China) by Hiperfect transfection reagent (QIAGEN) following the manufacturer's instructions, and all experiments were performed $24-48 \mathrm{~h}$ after transfection. The silencing efficiency siRNAs targeting IL13R $\alpha 1$ or DR 5 were measured by Western blot, and the two showing obviously silencing efficiencies were selected and combined at the equal concentration for following experiments. The sequences of siRNA targeting human IL13R $\alpha 1$ (silL13R $\alpha 1$ ) and human DR5 (siDR5) were as followings: silL13R $\alpha 1 \# 1$ : GGAAACTCGTCGTT CAATA; silL13R $\alpha 1 \# 2:$ GGAGCCAGCTCAAATTGTA; siDR5\#1: CAGCCGUAGUCUUGAUUGUUU; siDR5\#2: ACAAUCAAGACUACG GCUGUU. To evaluate the apoptosis of RA FLSs, RA FLSs were transfected with siRNA $(100 \mathrm{nM}), \mathrm{Lv}-\mathrm{IL} 13 \mathrm{R} \alpha 1$, or their respective controls in the presence of IL13, $\mathrm{CoCl}_{2}$, or Tm for $24 \mathrm{~h}$, respectively. Next, the cells were trypsinized and collected for detection of cell viability apoptosis with an Annexin V-FITC apoptosis detection kit according to the manufacturer's protocol.

\section{Cell viability}

RA FLSs were inoculated into a 96-well plate with 3000 cells/well, and three parallel duplicate wells were set in each group. After indicated treatments, $20 \mu \mathrm{L} /$ well MTS reaction solution was added at $24 \mathrm{~h}, 48 \mathrm{~h}$, and $72 \mathrm{~h}$, respectively. The culture dish was placed in an incubator with $5 \% \mathrm{CO}_{2}$ under $37^{\circ} \mathrm{C}$ to incubate $2 \mathrm{~h}$. The absorbance value (OD) was tested at a wavelength of $490 \mathrm{~nm}$ with an automatic microplate reader, and the growth curve was traced.

\section{Western blot and immunoprecipitation (IP)}

Cells were collected and lysed with RIPA buffer plus protease inhibitor cocktail (Roche Applied Science) [2]. For the chase assay of DR5 protein stability, CHX $(100 \mu \mathrm{g} / \mathrm{ml})$ was introduced for the indicated times. For IP, $10 \%$ of the lysate was set aside as the input control [2]. Cleared lysates were incubated with the indicated antibodies $(1 \mu \mathrm{g})$ and subsequent Protein A-coupled magnetic Dynabeads $(50 \mu \mathrm{l}$ from a stock solution; Thermo Fisher Scientific). Samples were subjected to standard SDS-PAGE, and the resulting bands were transferred onto polyvinylidene fluoride membrane for visualizing specific proteins [2].

Primary antibodies used in our study are described as followings: IL13R $\alpha 1$ (ab79277, Abcam), IL13R $\alpha 1$ (sc101382, Santa-Cruz Biotech), Trail (27,064, Proteintech), DR5 (ab199357, Abcam), ChoP (15,204, Proteintech), STAT6 (5397, Cell Signaling), phosphor-STAT6 (Tyr641) (5397, Cell Signaling), Bax (50,599, Proteintech), and Bcl (12,789, Proteintech). We used normal rabbit and mouse IgG (Santa Cruz Biotechnology, Inc.) as IP controls. The secondary antibodies used were HRP-coupled anti-mouse IgG and anti-rabbit IgG (GE Healthcare). Antibody binding was detected by enhanced chemiluminescence with hyperfilm ECL or an RGB 600 Imager (GE Healthcare).

\section{Quantitative real-time polymerase chain reaction (RT- qPCR) analysis}

RNA extraction and RT-qPCR analysis were performed as previously described [24]. We used the following primer sets: for human IL13R $\alpha 1$, 5-CGCGCCTACGGAAA CT CA-3 (forward) and 5-GGACCCCACTTGCAGACA A A-3 (reverse); human $\beta$-actin, 5-TCATTCCAAATATGAGATGCGTTGT-3 (forward) and 5-GCTATCACCT CCCCT GTGTG-3 (reverse); human trail, 5-TGCGTG CTGATCGTGATCTT-3 (forward) and 5-TCTTGGAG TCTTTCTAACGAGC (reverse). The analysis was performed using the $2^{-\Delta \Delta C T}$ method.

Immunohistochemistry (IHC) and immunofluorescence (IF) IHC was described as previously reported [24]. Briefly, the slides were incubated overnight with an anti-IL13R $\alpha 1$ antibody. For immunofluorescence, cells were sequentially probed with primary antibodies and fluorescent-labeled secondary antibodies (Jackson Immunoresearch, West Grove, PA). Images were captured under a confocal microscope (FV3000, OLYMPUS, Tokyo, Japan). 


\section{Histopathological examination}

Histopathological quantification was made by investigators in a blinded manner from images of three fields representing the distal interphalangeal joint, the proximal interphalangeal joint, and joints in the carpal region and averaged over all joints in one limb of each animal. Inflammation was scored 0 to 5 according to the following criteria: 0 , normal; 1 , minimal inflammatory infiltration; 2, mild infiltration; 3 , moderate infiltration with moderate edema; 4, marked infiltration with marked edema; and 5, severe infiltration with edema. Hyperplasia was defined as synovial tissue intimately invading the bone and/or cartilage and was scored 0 to 3 as follows: 0 , none; 1 , minimal; 2 , moderate; and 3 , severe. The bone degradation was scored using the following 0 to 3 scale: 0 , no bone erosion; 1, mild surface erosion; 2, moderate surface erosion; and 3, strong surface erosion. Sections were also stained by Safranin O/Fast green kit (Solarbio) for detection of cartilage damage (0: no changes; 1: erosion in part of the cartilage surface; 2: erosion of the cartilage surface and cartilage destruction; and 3: cartilage erosion and destruction, combined with a cartilage covered by connective tissue).

\section{GST pull-down assay and mass spectrometry (MS)}

Bacterially expressed GST-conjugated human IL13R $\alpha 1$ (GST- IL13R $\alpha 1$ ) or control GST (both $500 \mu \mathrm{g}$ ) bound to Glutathione-Sepharose 4B beads (Amersham Pharmacia, Piscataway, NJ, USA) was incubated with equal amounts of whole-cell lysates of RA FLSs at $4{ }^{\circ} \mathrm{C}$ for overnight. The washed complexes were then eluted by boiling in SDS sample buffer, separated by SDS-PAGE, and were incubated with anti-GST-conjugated beads for $24 \mathrm{~h}$ in a cold room. The immunoprecipitates were identified by mass spectrometry (BGI, China).

\section{Collagen-induced arthritis}

Chick collagen type II (CCII) $(2 \mathrm{mg} / \mathrm{mL}$; Chondrex, Inc., Redmond, WA, USA) was mixed with complete Freund's adjuvant (CFA) (Chondrex, Inc) and injected intradermally on day 0 at the base of the tail of with $100 \mu \mathrm{L}$ of an emulsion into 8- to 11-week-old DBA/1J mice. On day 21 , mice received an intradermal booster injection with $100 \mu \mathrm{g}$ of CCII in incomplete Freund's adjuvant (IFA) (Chondrex, Inc). To investigate the treatment efficacy of IL13R $\alpha 1$ at disease onset, Lv-mouse IL13R $\alpha 1$ or its control Lv-Ctrl with GFP tag was injected into the knee joints of $\mathrm{DAB} / 1 \mathrm{~J}$ mice. The thickness of the knee joints was measured using Vernier calipers. The clinical arthritis score was calculated as described previously [24]. The maximum possible score per mouse was 12 . Experiments were performed using 10 mice per group. Histological analysis of knee joints was fixed in $10 \%$ buffered formalin for $48 \mathrm{~h}$ and decalcified in 15\% EDTA.
The paws were then embedded in paraffin, and 5- $\mu \mathrm{m}$ sagittal serial sections of whole knee joints were cut. Tissue sections were stained with hematoxylin and eosin.

\section{Statistical analysis}

The arthritis scores, paw swelling, and cell viability were analyzed with the Mann-Whitney $U$ test as the requirement of Gaussian distribution and homogeneity of variance were not met. Differences in the levels of apoptosis, trail expression, apoptotic rate, and $\mathrm{Bax} / \mathrm{Bcl}$ ratio between the groups were analyzed with one-way ANOVA for homogeneity test of variance, and we fulfilled nonparametric Mann-Whitney $U$ test for the comparison between two groups due to the heterogeneity of variances. IL13R $\alpha 1$ expression levels at both mRNA and protein levels and histological score were confirmed to Gaussian distribution and homogeneity of variances and were compared with Student's unpaired $t$ test. Data were expressed as mean \pm standard deviation (SD) of the means. SPSS software (version 22.0; SPSS Inc., Chicago, IL, USA) was used to accomplish all statistical analysis. Two-sided $P$ values were considered as statistical significance if less than 0.05 .

\section{Results}

IL13Ra1 expression in the synovial tissues and fibroblasts from RA patients

To study the role of IL13R $\alpha 1$ in RA, its expression in the synovial tissues was firstly analyzed. The expression of IL13R $\alpha 1$ at both mRNA (Fig. 1a) and protein (Fig. 1b) levels were relatively lower in the synovial tissues from RA patients when compared to those from OA patients. Similar results of IL13R $\alpha 1$ staining was also duplicated by the IHC analysis (Fig. 1c, Supplementary Fig. 1a). In addition, IF analysis demonstrated that IL13R $\alpha 1$ was mainly localized in the cytoplasmic compartment of FLSs (Fig. 1d, Supplementary Fig. 1b). We also analyzed the expression of IL13R $\alpha 1$ in RA FLSs with the exposure of $\mathrm{CoCl}_{2}, \mathrm{Tm}$, or $\mathrm{Tg}$. Of them, $\mathrm{CoCl}_{2}$, as an inducer to mimic hypoxic condition, could also induce ER stress [25]. The data showed that IL13R $\alpha 1$ protein decreased obviously when RA FLSs were stimulated with $\mathrm{CoCl}_{2}, \mathrm{Tm}$, or $\mathrm{Tg}$ (Fig. $1 \mathrm{~d}$ and e). These results suggest that IL13R $\alpha 1$ may be involved in the cellular response of RA FLSs towards ER stress.

\section{Effect of IL13Ra1 on viability and apoptosis of RA FLSs}

In vitro experiments were then performed to investigate whether IL13R $\alpha 1$ could affect the biological activity of RA FLSs. Firstly, the cell viability increased more rapidly when IL13R $\alpha 1$ was silenced as compared to its negative control siCtrl (Fig. 2a). Moreover, the cell viability of RA FLSs decreased following the challenge of $\mathrm{Tm}$, Tg, and $\mathrm{CoCl}_{2}$. However, such reduction could be restored by silencing IL13R $\alpha 1$ (Fig. 2a). In contrast, the decrease of cell viability due to the challenge of $\mathrm{Tm}, \mathrm{Tg}$, and $\mathrm{CoCl}_{2}$ 


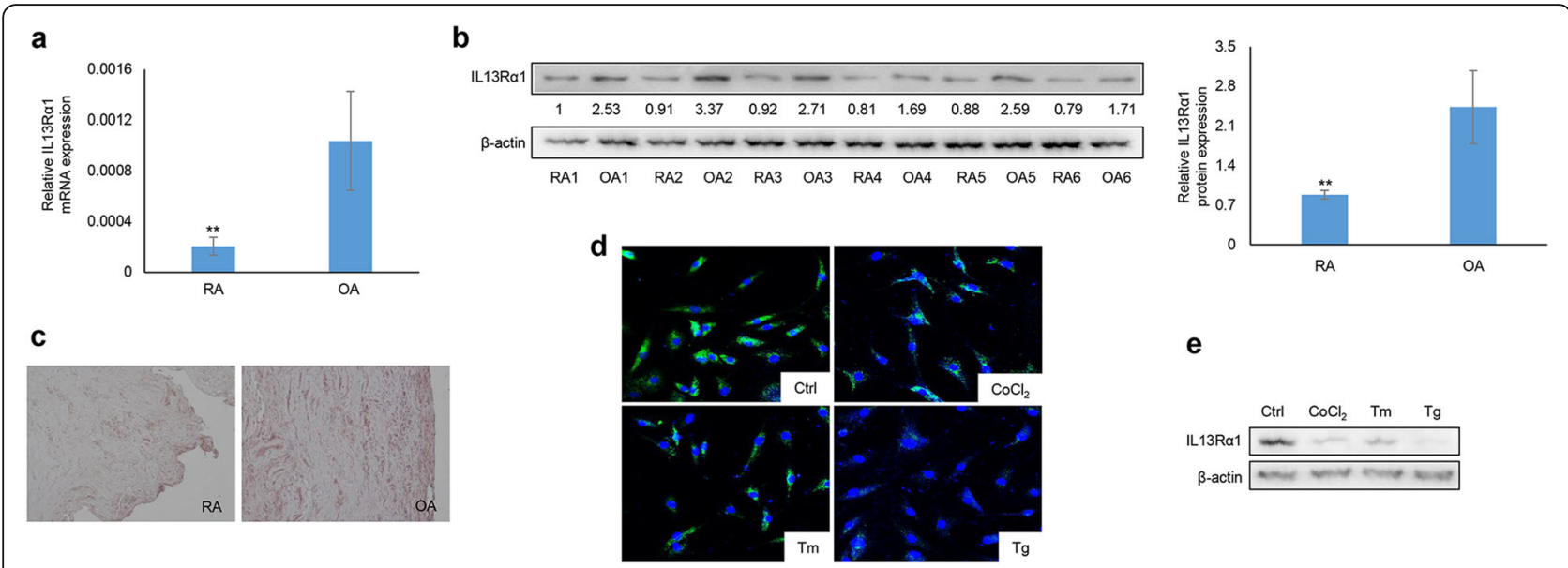

Fig. $1 \mathrm{IL} 13 \mathrm{Ra} 1$ is responsive to ER stress and promotes apoptosis of RA FLSs. a-c RT-qPCR, Western blot (WB), and IHC analyses of IL13Ra1 in the synovial tissues from RA $(n=12)$ and OA $(n=8)$ patients. Band intensity given underneath gel images was measured using Image J software ( $\mathrm{NIH}$, Bethesda, MD, USA) and presented as fold change compared with the first RA sample. d IF analysis of IL13Ra1 (green) in RA FLSS with the stimulation of Tunicamycin $(\mathrm{Tm}, 5 \mu \mathrm{g} / \mathrm{mL})$, Thapsigargin $(\mathrm{Tg}, 100 \mathrm{nM})$, or cobalt chloride $\left(\mathrm{CoCl}_{2}, 10 \mu \mathrm{M}\right)$ for $12 \mathrm{~h}$. e IB analyses of IL $13 \mathrm{Ra} 1 \mathrm{in} \mathrm{RA}$ FLSs with the stimulation of $\mathrm{Tm}, \mathrm{Tg}$, and $\mathrm{CoCl}_{2}$ as indicated doses for $12 \mathrm{~h}$. Data in $\mathbf{d}$ and $\mathbf{e}$ represent three independent experiments from three different RA samples with similar results. ${ }^{* *} p<0.01$ compared with RA

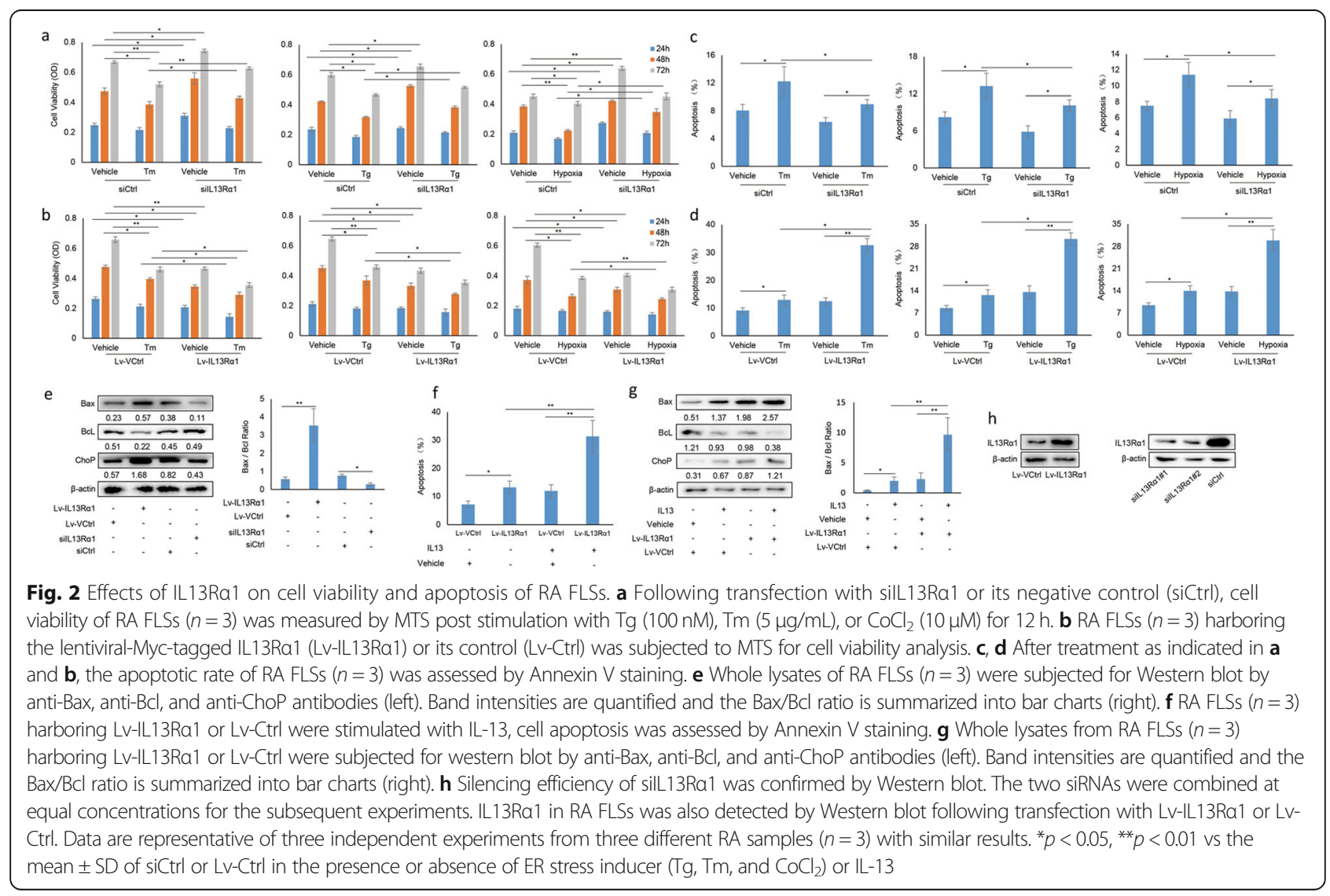


became more obvious when IL13R $\alpha 1$ was overexpressed by lentiviral transfection (Lv-IL13R $\alpha 1)$ as compared with its vector control (Lv-Ctrl) (Fig. 2b).

Then, we analyzed the apoptosis of RA FLSs when IL13R $\alpha 1$ expression was silenced or overexpressed. The data showed that the apoptotic proportions of RA FLSs decreased (Fig. 2c) or increased (Fig. 2d) respectively, when IL13R $\alpha 1$ was silenced or overexpressed as compared to their parental control ones. Additionally, the increase of apoptotic RA FLSs due to $\mathrm{CoCl}_{2}, \mathrm{Tm}$, or $\mathrm{Tg}$ challenge could be attenuated by silencing IL13R $\alpha 1$ (Fig. 2c), but became more obvious by overexpressing it (Fig. 2d). Bax or Bcl-2 represents pro-apoptotic or anti-apoptotic markers and the $\mathrm{Bax} / \mathrm{Bcl}-2$ ratio serves to determine cell susceptibility to apoptosis [26]. Further analysis by Western blot supported that the $\mathrm{Bax} / \mathrm{Bcl}-2$ ratio decreased significantly in RA FLSs when IL13R $\alpha 1$ was knocked down, but enhanced more obviously when IL13R $\alpha 1$ was overexpressed (Fig. 2e).
In this study, we also analyzed the effect of IL13R $\alpha 1$ on cell apoptosis in the presence of its ligand, IL-13. Although IL-13 treatment induced a marginal increase of the apoptotic rate in RA FLSs, such an increase became more obvious by overexpressing IL $13 R \alpha 1$ simultaneously (Fig. 2f). A similar increase of $\mathrm{Bax} / \mathrm{Bcl}-2$ ratio was also found when IL-13-stimulated RA FLSs were overexpressed by IL13R $\alpha 1$ (Fig. 2g). However, no obvious increase of apoptosis was observed when IL13R $\alpha 1$ was overexpressed in OA FLSs (Supplementary Fig. 2). Furthermore, the expression of IL13R $\alpha 1$ in RA FLSs following transfection with silL13R $\alpha 1 /$ siCtrl or Lv-IL13R $\alpha 1 /$ Lv-Ctrl were shown in Fig. $2 \mathrm{~h}$.

\section{IL13Ra1 promotes cell apoptosis of RA FLSs through upregulating trail}

Trail-DR5-mediated signaling is important for the apoptosis of RA FLSs [15]. In this study, we found that the expression of the trail in RA FLSs decreased when IL13R $\alpha 1$ was silenced (Fig. 3a), but increased when

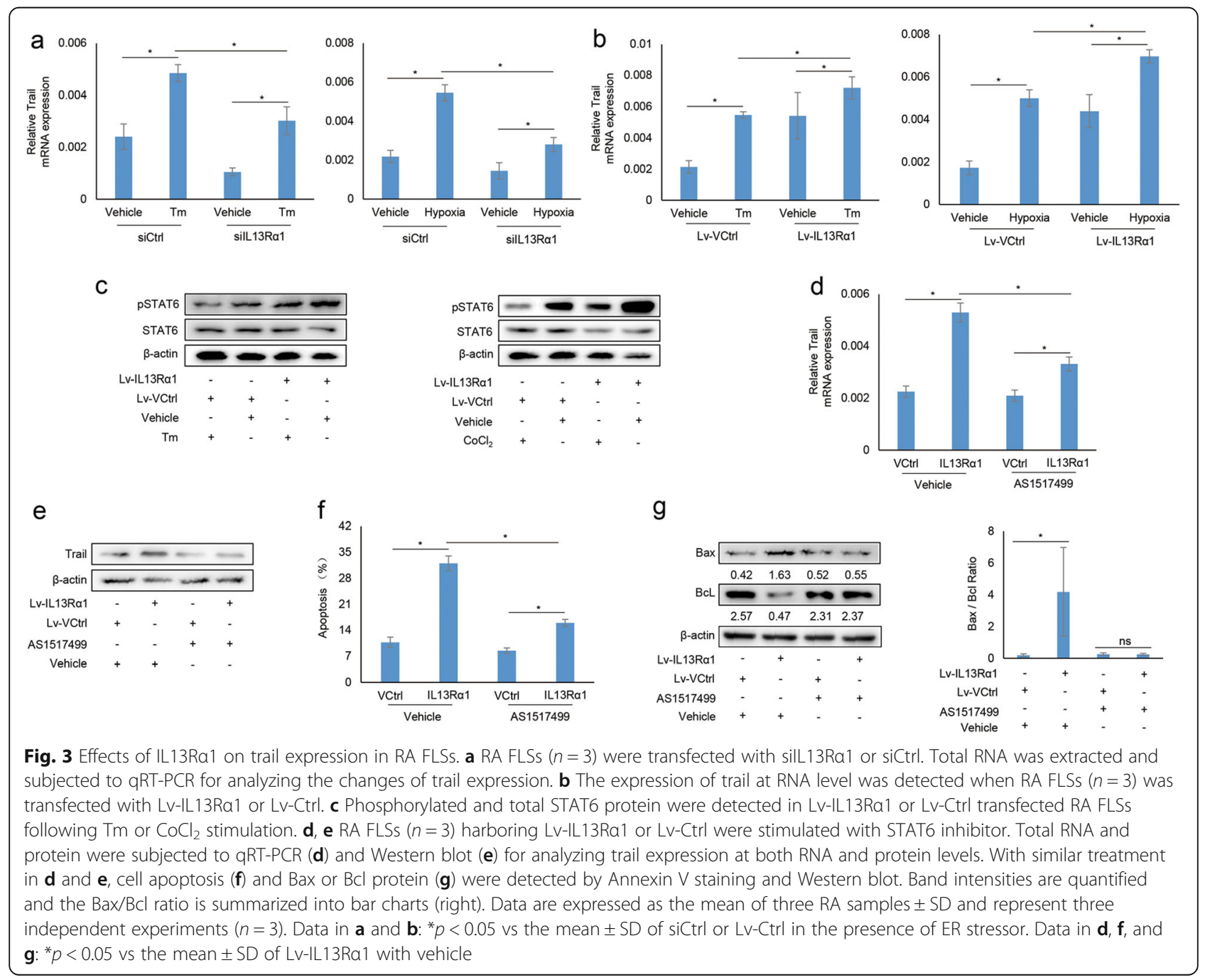


IL13R $\alpha 1$ was overexpressed (Fig. 3b) as compared with their parental control ones. IL13R $\alpha 1$ was reported that it could activate STAT6 after binding to IL13 [8]. Here, our data showed that the phosphorylated levels of STAT6, but not its total protein levels, increased in RA FLSs when IL13R $\alpha 1$ was overexpressed (Fig. 3c), and such increase was still obvious when RA FLSs were stimulated by Tm or $\mathrm{CoCl}_{2}$ simultaneously. To determine whether IL13R $\alpha 1$ regulated trail expression through STAT6, Lv-IL13R $\alpha 1$-, or Lv-Ctrl-transfected FLSs was treated with STAT6 inhibitor, AS1517499. The data showed that AS1517499 treatment could attenuate the increase of the trail resulting from IL13R $\alpha 1$ overexpression (Fig. 3d and e). Furthermore, the increase of apoptotic rate (Fig. 3f) and the Bax/ $\mathrm{BcL}$ ratio (Fig. 3g) resulting from IL13R $\alpha 1$ overexpression could be reverted when RA FLSs were treated with AS1517499. These results suggest that IL13R $\alpha 1$ induces the expression of trail via STAT6.
IL13Ra1 interacts and stabilizes DR5 protein in RA FLSs To further elucidate the mechanism of IL13R $\alpha 1$ in RA, GST pull-down plus mass spectrum was applied. Among its potential interactors (Supplementary Table 2), DR5 was selected for further analysis as it is closely related to cell apoptosis. Our data showed that IL13R $\alpha 1$ and DR5 reciprocally interacted in RA FLSs and their coprecipitation efficacy increased when RA FLSs were introduced by IL13R $\alpha 1$ in the presence of $\mathrm{CoCl}_{2}$ or Tm (Fig. 4a). Co-localization of IL13R $\alpha$ and DR5 was also confirmed by IF analysis (Fig. 4b). Furthermore, with the stimulation of $\mathrm{CoCl}_{2}$ or Tm, IL13R $\alpha 1$ overexpression upregulated (Fig. 4c), while silencing IL13R $\alpha 1$ downregulated the expression levels of DR5 protein in RA FLSs (Fig. 4d). Detailed analysis showed that DR5 protein degraded more slowly in IL13R $\alpha 1$-overexpressing RA FLSs when compared with its control (Fig. 4e). However, with the stimulation of $\mathrm{CoCl}_{2}$, DR5 protein degraded more rapidly in silL13R $\alpha 1$-transfected RA FLSs when
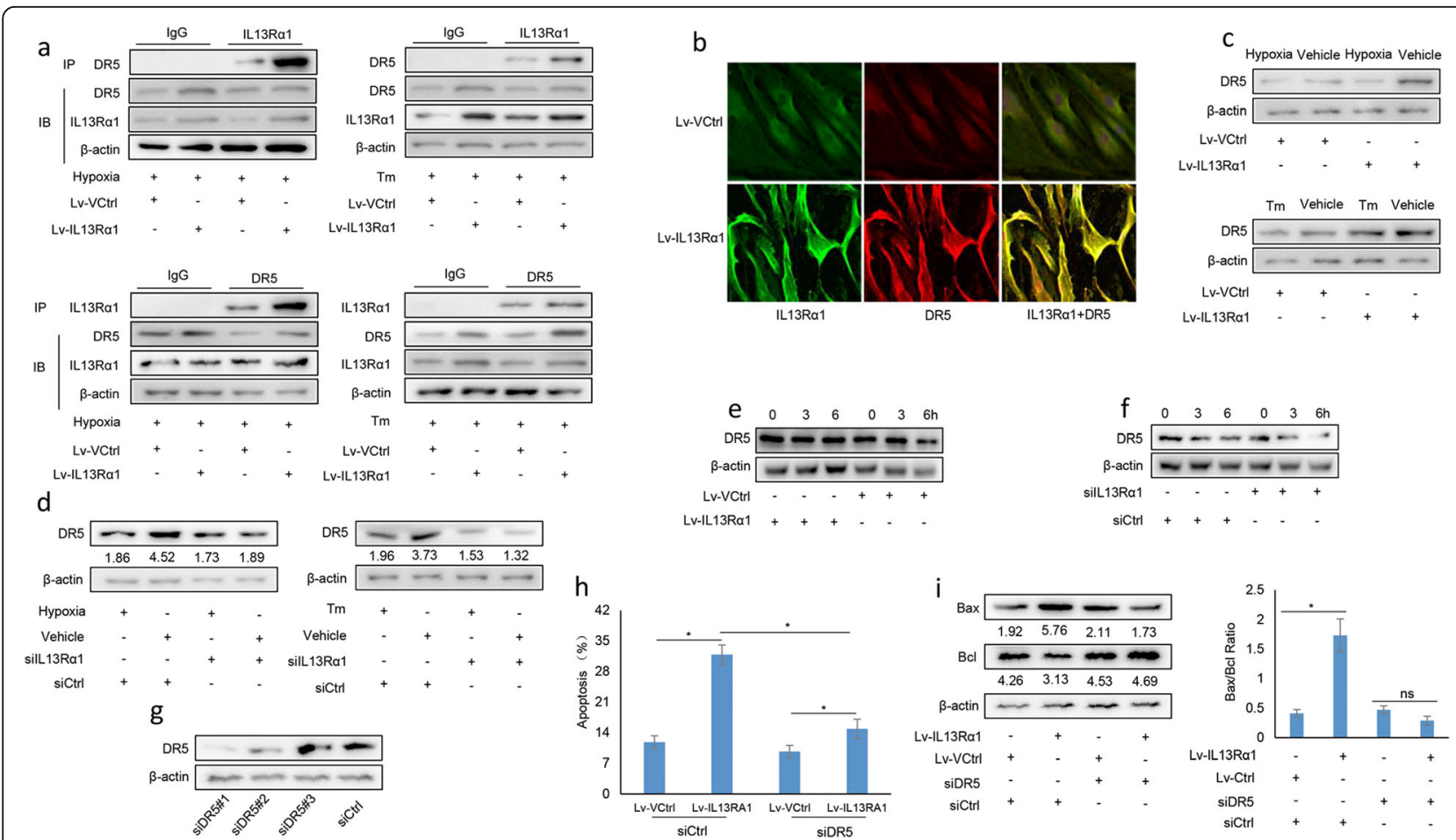

Fig. 4 IL13Ra1 interacts and stabilizes DR5. a Whole lysates from $\mathrm{CoCl}_{2}$ or Tm challenged RA FLSs were IP with anti-IL13Ra1 or anti-DR5 and IB by anti-DR5 or anti-IL13Ra1. b After transfection with Lv-IL13Ra1 or Lv-Ctrl for $24 \mathrm{~h}$, IL13Ra1 (green) and DR5 (red) in RA FLSs from three samples were detected by IF. $\mathbf{c}$ Western blot of DR5 protein in RA FLSs following transfection with LV-IL13Ra1 relative to Lv-Ctrl. d With the exposure of $\mathrm{CoCl}_{2}$ or Tm, RA FLSs were transfected with silL13Ra1/siCtrl for $24 \mathrm{~h}$, DR5 protein was measured by Western blot, and band intensity given underneath gel images was measured using ImageJ software. e, $\mathbf{f}$ With the exposure of CHX plus Tm, total proteins from Lv-IL13Ra1/Lv-Ctrltransfected or silL13Ra1/siCtrl-transfected RA FLSs were subjected to Western blot for analyzing DR5 expression as indicated time points. Band intensity was measured using ImageJ software and presented as fold change compared as indicated. g Silencing efficiency of siRNA targeting DR5 was detected by Western blot, and these two siRNAs were combined at an equal concentration for the following experiments. RA FLSs $(n=$ 3) harboring LV-IL13Ra1 or LV-Ctrl were transfected with siDR5 or siCtrl, and the apoptotic rate (h) and Bax or Bcl protein (i) were detected by Annexin $\vee$ staining and western blot. Band intensities are quantified and the Bax/Bcl ratio is summarized into bar charts (right). Data are representative of three independent experiments from three different RA samples $(n=3)$ with similar results. ns, not significant vs the mean \pm SD of $\mathrm{Lv}$-Ctrl in the presence of siDR5; ${ }^{*} p<0.05$ vs the mean \pm SD of IL13Ra1 in the presence of siCtrl 
compared with siCtrl-transfected ones (Fig. 4f). To further elucidate the involvement of DR5 in the proapoptotic effect of IL13R $\alpha 1$, siRNA targeting DR5 was applied in RA FLSs with IL13R $\alpha 1$ overexpression, and the silencing efficiency of DR5 was confirmed by Western blot (Fig. 4g). We found that the induction of apoptosis resulting from IL13R $\alpha 1$ overexpression, as evidenced by the increase of Annexin $\mathrm{V}$ staining and Bax/Bcl-2 rate, could be attenuated by silencing DR5 in RA FLSs (Fig. 4h and i). Thus, DR5 was also involved in the pro-apoptotic effect of IL13R $\alpha 1$.

\section{IL13Ra1 ameliorate the arthritis phenotype of RA}

To assess the therapeutic potential of IL13R $\alpha 1$, CIA models were treated with Lv-IL13R $\alpha 1$ and Lv-Ctrl $\left(10^{9}\right.$ PFU per joint) and the study period extended up to 21 days post injection. The presence of GFP in the synovial tissues as evidenced by Western blot analysis confirmed the transfection efficiency of Lv-IL13R $\alpha 1$ and Lv-Ctrl (Fig. 5a). The results show that the articular index (Fig. 5b) and knee joint thickness (Fig. 5c) were obviously ameliorated post-intra-articular injection of LvIL13R $\alpha 1$ when compared with its controls. Histological analysis of the joints revealed less degree of synovial hyperplasia, inflammation, cartilage destruction, and bone erosion with Lv-IL13R $\alpha 1$ injection (Fig. 5d and e). To confirm such therapeutic effect of Lv-IL13R $\alpha 1$ injection was achieved by direct targeting FLSs, our data confirmed that, besides the increase of IL13R $\alpha 1$, DR5, and Trail, Bax/Bcl ratio increased significantly as well in synovial tissues from Lv-IL13R $\alpha 1$ injected ones (Fig. 5a). FLSs were also freshly isolated from the CIA models and the apoptotic rate increased significantly in FLSs when introducing Lv-IL13R $\alpha 1$ in contrast to Lv-Ctrl (Fig. 5f). Collectively, these data support that IL13R $\alpha 1$ plays an anti-arthritis role in RA.

\section{Discussion}

Previous study demonstrated that ER stress-associated gene signatures are highly expressed in RA synovium [6]. In addition, GRP78 and Synoviolin could prevent RA FLSs against ER stress-induced apoptosis and contribute to the chronic inflammation and joint destruction $[3,6,27]$. Thus, identifying key factors accounting for the apoptotic resistance of RA FLSs against ER stress would hold great promise for RA intervention. Previous study demonstrated that IL13R $\alpha 1$ regulates the cellular responses to various stressful stimulations that it could promote more fibrosis in response to transforming growth factor- $\beta$ (TGF- $\beta$ ) [28]. Likewise, neuronal IL13R $\alpha 1$ increases the susceptibility to oxidative damage [29]. In this study, our loss- and gain- of functional experiments argued IL13R $\alpha 1$-mediated signaling pathway as a novel molecular program to counter the apoptotic resistance of RA FLSs towards ER stress. Integrating the data obtained from the in vitro studies about ER stresschallenged RA FLSs and the in vivo experiments that transduced IL13R $\alpha 1$ into CIA models enabled us to deduce that targeting IL13R $\alpha 1$ may provide novel treatment strategy for RA. In addition, the relatively lower levels of IL13R $\alpha 1$ expression at steady state in RA FLSs and the further decrease in response to ER stress may explain the reason why RA FLSs are apoptosis resistance against ER stress.

Although the exact roles of IL-13 in RA were paradoxical from previous studies [9-11], in this study, we

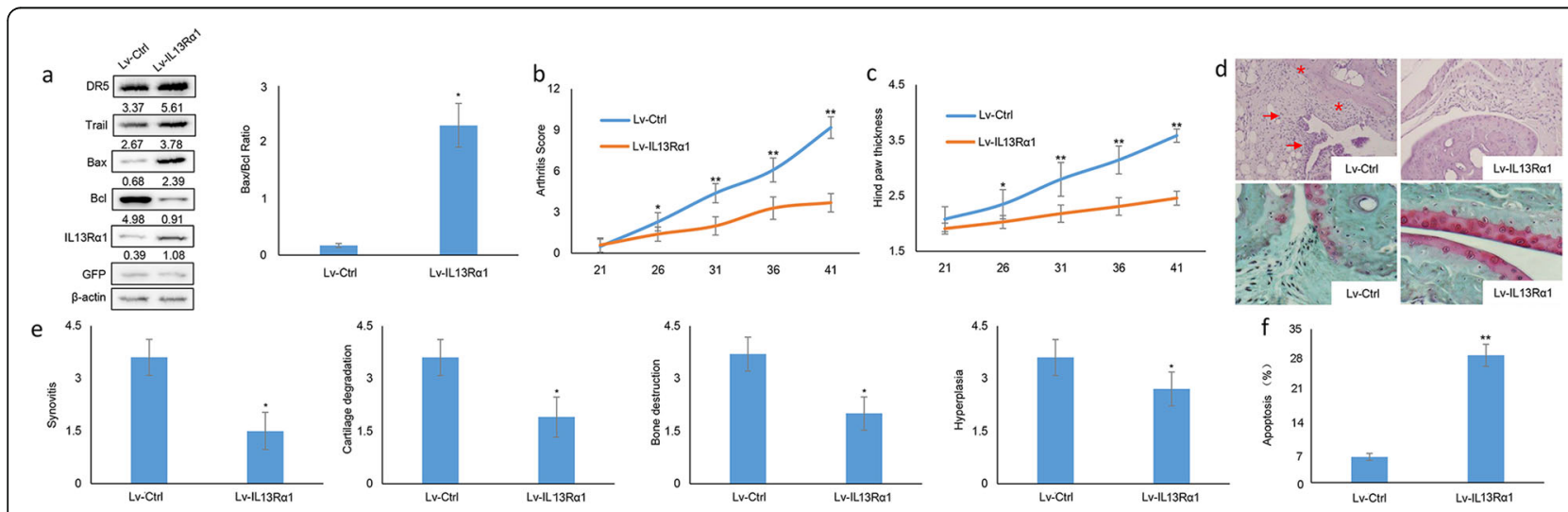

Fig. 5 Treatment with IL13Ra1 halts and reverses the development of CIA. a The synovial tissues from knee joints in LV-IL13Ra1 or LV-Ctrl transfected CIA mice ( $n=10$ per group) at the resolution phase of arthritis were homogenized in cell lysis buffer for Western blot detection of IL13Ra1, DR5, Trail, Bax, Bcl, and GFP (left). Band intensities are quantified and the Bax/Bcl ratio is summarized into bar charts (right). b, c Comparison of the clinical score $(\mathbf{b})$ and paw swelling (c) between arthritic mice treated with LV-Myc-IL13Ra1 ( $n=10)$ or LV-Ctrl $(n=10)$. $\mathbf{d}$ Representative images of hematoxylin-eosin (H\&E, upper) and Safranin O/Fast green staining (lower) of the knee joint. The synovial thickening (red arrow) and bone erosion (red asterisk) are shown $(n=10)$. e Inflammation, hyperplasia, cartilage degradation, and bone destruction as evaluated through the scoring system ( $n=10$ per group). f FLSs were isolated from knee joints of CIA models ( $n=6$ per group) and subjected to flow cytometry for analyzing apoptosis. ${ }^{*} p<0.001$ vs the mean \pm SD of indicated parameters in Lv-Ctrl 
confirmed the anti-inflammatory role of IL13 in RA that it requires IL13R $\alpha 1$ to induce apoptosis of RA FLSs. Additionally, we also defined the molecular mechanism underlying how IL13R $\alpha 1$ combats the apoptotic resistance of RA FLSs against ER stress that IL13R $\alpha 1$ could activate Trail-DR5 signaling pathway through upregulating the expression of the trail via activating STAT6 and simultaneously interact and stabilize the receptor of the trail, DR5. More importantly, IL13R $\alpha 1$ overexpression could promote cell apoptosis even in the absence of its ligand IL13 and ER stress inducer. These facts indicate that IL13R $\alpha 1$ acts as a scaffold in the membrane to activate DR5 and STAT6, respectively, and the change of IL13R $\alpha 1$ level (quantity) is more important other than its ligands to regulate cell apoptosis.

As a TNF superfamily member, trail protects against the inflammation progression of RA [30]. Furthermore, the local administration of trail inhibits the development of arthritis, whereas the blockade of trail-mediated apoptosis increases the occurrence and severity of arthritis in CIA models [31]. However, the application of the trail as a therapeutic agent might be questioned because of the ability of the soluble trail to induce apoptosis of normal hepatocytes [32]. Here, our data showed that the proapoptotic effects of IL13R $\alpha 1$ on OA FLSs are not as obvious as those on RA FLSs, indicating the diseasespecific role of IL13R $\alpha 1$ in RA. Although our in vivo experiments showed that IL13R $\alpha 1$ could be transferred into synovial tissues by lentiviral vector and thus conferred synovial tissues with a pro-apoptotic feature, we could not exclude the possibility that T cells, B cells, and monocytes/ macrophages, which are involved in immune-mediated inflammation, might also be targets for IL13R $\alpha 1$-mediated apoptosis.

\section{Conclusion}

Together, our findings provide an alternative option to overcome apoptosis resistance to ER stress of RA FLSs and a novel treatment strategy for RA.

\section{Supplementary information}

Supplementary information accompanies this paper at https://doi.org/10. 1186/s13075-020-02270-4.

Additional file 1: Fig. S1. Negative control for IHC and IF analysis. (a) Synovial tissues from RA and OA patients were subjected to IHC analysis with primary antibody against IgG instead of IL13Ra1. (b) RA FLSs with the stimulation of $\mathrm{CoCl}_{2}, \mathrm{Tm}$ and $\mathrm{Tg}$ were subjected to $\mathrm{IF}$ analysis with primary antibody against lgG instead of IL13Ra1. (c) RA FLSs transfected with LV-Myc-IL13Ra1 or Lv-Ctrl were subjected to co-focal immunofluorescent analysis with primary antibody against IgG instead of IL13Ra1 or DR5.

Additional file 2: Fig. S2. Effects of IL13Ra1 on apoptosis of OA FLSS. FLSs were isolated from OA ( $n=3$ per group) and subjected to flow cytometry for analyzing apoptosis. ns, not significant vs the mean \pm SD of indicated parameters in Lv-Ctrl.
Additional file 3: Table S1 Clinical characteristics of RA patients.

Additional file 4: Table S2 List of potential IL13Ra1 partners in RA FLSs identified by mass spectrometry.

\begin{abstract}
Abbreviations
RA: Rheumatoid arthritis; FLSs: Fibroblast-like synoviocytes; ER: Endoplasmic reticulum; UPR: Unfolded protein response; Tg: Thapsigargin;

Tm: Tunicamycin; siRNA: Small interfering RNA; IP: Immunoprecipitation; RTQPCR: Quantitative real-time polymerase chain reaction;

IHC: Immunohistochemistry; IF: Immunofluorescence; MS: Mass spectrometry; CCII: Collagen type II; CFA: Complete Freund's adjuvant; IFA: Incomplete Freund's adjuvant; TGF- $\beta$ : Transforming growth factor- $\beta$; STAT6: Transducer and activator of transcription 6; CIA: Collagen-induced arthritis; $\mathrm{CoCl}_{2}$ : Cobalt chloride 2; DMEM: Dulbecco's modified Eagle's medium
\end{abstract}

\section{Acknowledgements}

Not applicable.

\section{Authors' contributions}

G Song and L Wang conceived the study; L Qiao, L Tian, and T Xie collected the clinical samples; X Yang, Q Guo, T Feng, Q Lu, L Ge, J Pan, K Bi, and C Yao performed experiments; $X$ Yang, $G$ Song, and $L$ Wang analyzed data and wrote the manuscript. The authors read and approved the final manuscript.

\section{Funding}

This work was supported by the National Natural Science Foundation of China (Grant No. 81772760), The Shandong Taishan Scholarship (NO. tsqn20161076), National Science and Technology Major Project (2017ZX09301030), The Innovation Project of Shandong Academy of Medical Sciences, The Youth Innovation Technology Plan of Shandong University (2019KJK003), and the academic promotion program of the Shandong First Medical University (LJ001).

\section{Availability of data and materials}

The datasets used and analyzed during the current study are available from the corresponding author on reasonable request.

\section{Ethics approval and consent to participate}

Synovial tissues used in this study were provided by the Shandong Provincial Hospital and Shandong Qianfo Mountain Hospital. Tissues were collected during knee replacement surgery from patients with RA and OA. Participants provided written informed consent to participate in the study and to allow their biological samples to be genetically analyzed. The Ethical Committee of the Shandong Academy of Medical Sciences approved this study.

\section{Consent for publication}

Not applicable.

\section{Competing interests}

The authors declare that they have no competing interests.

\section{Author details}

'Department of Hematology, Qilu Children's Hospital of Shandong University, Jinan, China. ${ }^{2}$ Shandong Provincial Qianfoshan Hospital, Shandong University, Jinan, China. ${ }^{3}$ Department of Pathology, Shandong University Medical School, Jinan, China. ${ }^{4}$ Department of Rheumatology and Autoimmunology, The First Affiliated Hospital of Shandong First Medical University, Key Lab for Biotech-Drugs of National Health Commission, Key Lab for Rare \& Uncommon Diseases of Shandong Province, Shandong Medicinal Biotechnology Centre, Jinan 250062, China. ${ }^{5}$ School of Medicine and Life Sciences, University of Jinan-Shandong Academy of Medical Sciences, Jinan, China. ${ }^{6}$ Department of Joint Surgery, Shandong Provincial Hospital Affiliated to Shandong University, Jinan, China. ${ }^{7}$ Department of Rheumatology, Shandong Provincial Hospital Affiliated to Shandong University, Jinan, China. Institute of Basic Medicine, Shandong First Medical University \& Shandong Academy of Medical Sciences, Jinan 250062, China. 
Received: 20 November 2019 Accepted: 13 July 2020

Published online: 08 August 2020

\section{References}

1. Rahmati M, Moosavi MA, McDermott MF. ER stress: a therapeutic target in rheumatoid arthritis? Trends Pharmacol Sci. 2018;39:610-23.

2. Wang L, Dong H, Song G, Zhang R, Pan J, Han J. TXNDC5 synergizes with HSC70 to exacerbate the inflammatory phenotype of synovial fibroblasts in rheumatoid arthritis through NF-kappaB signaling. Cell Mole Immunol. 2018; 15:685-96.

3. Park YJ, Yoo SA, Kim WU. Role of endoplasmic reticulum stress in rheumatoid arthritis pathogenesis. J Korean Med Sci. 2014;29:2-11.

4. Zhang K, Kaufman RJ. From endoplasmic-reticulum stress to the inflammatory response. Nature. 2008;454:455-62.

5. Kabala PA, Angiolilli C, Yeremenko N, Grabiec AM, Giovannone B, Pots D, et al. Endoplasmic reticulum stress cooperates with Toll-like receptor ligation in driving activation of rheumatoid arthritis fibroblast-like synoviocytes. Arthritis Research Therapy. 2017;19:207.

6. Yoo SA, You S, Yoon HJ, Kim DH, Kim HS, Lee K, et al. A novel pathogenic role of the ER chaperone GRP78/BiP in rheumatoid arthritis. J Exp Med. 2012;209:871-86.

7. Yamasaki S, Yagishita N, Tsuchimochi K, Kato Y, Sasaki T, Amano T, et al. Resistance to endoplasmic reticulum stress is an acquired cellular characteristic of rheumatoid synovial cells. Int J Mol Med. 2006;18:113-7.

8. Hershey GK. IL-13 receptors and signaling pathways: an evolving web. J Allergy Clin Immunol. 2003:111:677-90.

9. Mao YM, Zhao CN, Leng J, Leng RX, Ye DQ, Zheng SG, et al. Interleukin-13: a promising therapeutic target for autoimmune disease. Cytokine Growth Factor Rev. 2019;45:9-23.

10. Woods JM, Amin MA, Katschke KJ Jr, Volin MV, Ruth JH, Connors MA, et al. Interleukin-13 gene therapy reduces inflammation, vascularization, and bony destruction in rat adjuvant-induced arthritis. Hum Gene Ther. 2002;13:381-93.

11. Woods JM, Haines GK, Shah MR, Rayan G, Koch AE. Low-level production of interleukin-13 in synovial fluid and tissue from patients with arthritis. Clin Immunol Immunopathol. 1997;85:210-20.

12. Karo-Atar D, Bordowitz A, Wand O, Pasmanik-Chor M, Fernandez IE, Itan M, et al. A protective role for IL-13 receptor alpha 1 in bleomycin-induced pulmonary injury and repair. Mucosal Immunol. 2016;9:240-53.

13. Liu SH, Yang CN, Pan HC, Sung YJ, Liao KK, Chen WB, et al. IL-13 downregulates PPAR-gamma/heme oxygenase-1 via ER stress-stimulated calpain activation: aggravation of activated microglia death. Cell Mole Life Sci. 2010;67:1465-76

14. Arensdorf AM, Rutkowski DT. Endoplasmic reticulum stress impairs IL-4/IL-13 signaling through C/EBPbeta-mediated transcriptional suppression. J Cell Sci. 2013;126:4026-36.

15. Chaudhary PM, Eby M, Jasmin A, Bookwalter A, Murray J, Hood L. Death receptor 5, a new member of the TNFR family, and DR4 induce FADDdependent apoptosis and activate the NF-kappaB pathway. Immunity. 1997; 7:821-30.

16. Walczak H, Degli-Esposti MA, Johnson RS, Smolak PJ, Waugh JY, Boiani N, et al. TRAIL-R2: a novel apoptosis-mediating receptor for TRAIL. EMBO J. 1997; 16:5386-97.

17. Pan G, O'Rourke K, Chinnaiyan AM, Gentz R, Ebner R, Ni J, et al. The receptor for the cytotoxic ligand TRAIL. Science. 1997;276:111-3.

18. Pan G, Ni J, Wei YF, Yu G, Gentz R, Dixit VM. An antagonist decoy receptor and a death domain-containing receptor for TRAlL. Science. 1997;277:815-8.

19. Marsters SA, Sheridan JP, Pitti RM, Huang A, Skubatch M, Baldwin D, et al. A novel receptor for Apo2L/TRAIL contains a truncated death domain. Current Biol. 1997;7:1003-6.

20. Emery JG, McDonnell P, Burke MB, Deen KC, Lyn S, Silverman C, et al. Osteoprotegerin is a receptor for the cytotoxic ligand TRAIL. J Biol Chem. 1998;273:14363-7

21. Ichikawa K, Liu W, Fleck M, Zhang H, Zhao L, Ohtsuka T, et al. TRAlL-R2 (DR5) mediates apoptosis of synovial fibroblasts in rheumatoid arthritis. $J$ Immunol. 2003;171:1061-9.

22. Arnett FC, Edworthy SM, Bloch DA, McShane DJ, Fries JF, Cooper NS, et al. The American Rheumatism Association 1987 revised criteria for the classification of rheumatoid arthritis. Arthritis Rheum. 1988;31:315-24.

23. Altman $R$, Asch $E$, Bloch D, Bole G, Borenstein D, Brandt $K$, et al. Development of criteria for the classification and reporting of osteoarthritis. Classification of osteoarthritis of the knee. Diagnostic and Therapeutic
Criteria Committee of the American Rheumatism Association. Arthritis Rheum. 1986;29:1039-49.

24. Wang $L$, Zheng $Y, X u H$, Yan $X$, Chang $X$. Investigate pathogenic mechanism of TXNDC5 in rheumatoid arthritis. PLoS One. 2013;8:e53301.

25. Song W, Sheng L, Chen F, Tian Y, Li L, Wang G, et al. C. Sakazakii activates AIM2 pathway accompanying with excessive ER stress response in mammalian mammary gland epithelium. Cell Stress Chaperones. 2020;25: 223-33.

26. López-Hernández B, Ceña V, Posadas I. The endoplasmic reticulum stress and the HIF-1 signalling pathways are involved in the neuronal damage caused by chemical hypoxia. Br J Pharmacol. 2015;172:2838-51.

27. Gao B, Lee SM, Chen A, Zhang J, Zhang DD, Kannan K, et al. Synoviolin promotes IRE1 ubiquitination and degradation in synovial fibroblasts from mice with collagen-induced arthritis. EMBO Rep. 2008;9:480-5.

28. Rothenberg ME, Wen T, Shik D, Cole ET, Mingler MM, Munitz A. IL-13 receptor alpha1 differentially regulates aeroallergen-induced lung responses. J Immunol. 2011;187:4873-80.

29. Mori S, Sugama S, Nguyen W, Michel T, Sanna MG, Sanchez-Alavez M, et al. Lack of interleukin-13 receptor alpha1 delays the loss of dopaminergic neurons during chronic stress. J Neuroinflammation. 2017;14:88.

30. Wiley SR, Schooley K, Smolak PJ, Din WS, Huang CP, Nicholl JK, et al. Identification and characterization of a new member of the TNF family that induces apoptosis. Immunity. 1995;3:673-82.

31. Song K, Chen Y, Goke R, Wilmen A, Seidel C, Goke A, et al. Tumor necrosis factor-related apoptosis-inducing ligand (TRAIL) is an inhibitor of autoimmune inflammation and cell cycle progression. J Exp Med. 2000;191: 1095-104.

32. Jo M, Kim TH, Seol DW, Esplen JE, Dorko K, Billiar TR, et al. Apoptosis induced in normal human hepatocytes by tumor necrosis factor-related apoptosis-inducing ligand. Nat Med. 2000;6:564-7.

\section{Publisher's Note}

Springer Nature remains neutral with regard to jurisdictional claims in published maps and institutional affiliations.

Ready to submit your research? Choose BMC and benefit from:

- fast, convenient online submission

- thorough peer review by experienced researchers in your field

- rapid publication on acceptance

- support for research data, including large and complex data types

- gold Open Access which fosters wider collaboration and increased citations

- maximum visibility for your research: over $100 \mathrm{M}$ website views per year

At $\mathrm{BMC}$, research is always in progress.

Learn more biomedcentral.com/submissions 